\title{
Directional Anisotropy of Swift Gamma-Ray Bursts
}

\author{
Péter Veres ${ }^{*, \dagger}$, Zsolt Bagoly*, István Horváth ${ }^{\dagger}$, Lajos G. Balázs**, Attila \\ Mészáros ${ }^{\ddagger}$ and János Kelemen** \\ ${ }^{*}$ Eötvös University, Budapest \\ ${ }^{\dagger}$ Bolyai Military University, Budapest \\ ${ }^{* *}$ Konkoly Observatory, Budapest \\ ${ }^{\ddagger}$ Charles University, Prague
}

\begin{abstract}
Swift satellite measurements contributed substantially to the gamma-ray burst (GRB) redshift observations through fast slewing to the source of the GRBs. Still, a large number of bursts are without redshift. We study the celestial distribution of bursts with various methods and compare them to a random catalog using Monte-Carlo simulations. We find an anisotropy in the distribution of the intermediate class of bursts and find that the short and long population are distributed isotropically.
\end{abstract}

Keywords: gamma-rays: bursts, methods: statistical

PACS: 95.55.Ka, 95.85.Pw, 97.10.Yp, 98.62.Py, 98.62.Ve

\section{INTRODUCTION}

Swift-BAT can observe 1.4 sr of the sky at any given moment. Its field of view is not uniform in sensitivity. We search for directional anisotropies of the GRBs using coordinatesystem independent tests. To compute the significance of the tests we perform MonteCarlo simulations for the short, intermediate and long populations of the bursts taking into account the exposure function of Swift. The group memberships are calculated on the hardness-duration plane using a modified maximum likelihood method [1]. While the exposure map of BATSE [2] depends only on the declination, Swift has a more complicated exposure function. To deal with this, we used the HEALPix [3] pixelization algorithm.

\section{EXPOSURE FUNCTION}

Because Swift makes pointed observations, its sky sensitivity map will be dependent on many factors. We place the Swift sensitivity mask on the celestial sphere centered on the $(\alpha, \delta)$ coordinates in the catalog. We rotate the mask with the appropriate angle as indicated by the Roll_angle parameter. Then we multiply this with the exposure time spent on that location. We carry out this exercise for all observations and sum up the results. The exposure map on Fig. 1 shows a paucity of exposure time in the direction of the ecliptic, and a variation of roughly a factor of two between the extremes. It also shows a similarity in structure with the 22 month exposure map of Tueller J. et al. [4]. 

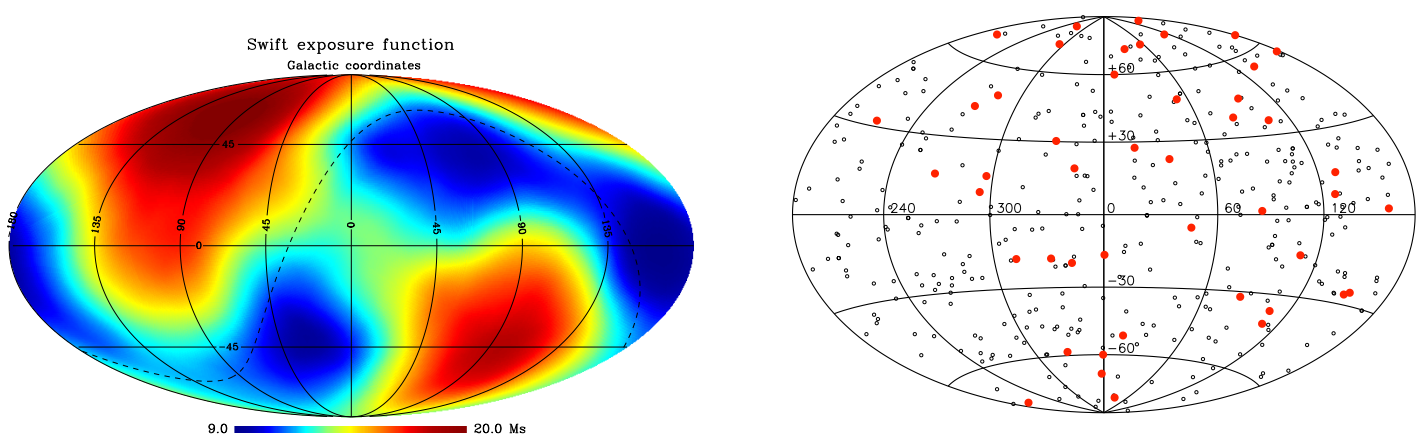

FIGURE 1. Figures showing the Swift exposure function in Mollweide projection in Galactic coordinates(left). The dotted line shows the ecliptic plane. The celestial distribution of the intermediate group (filled red circles) and the total sample (empty black circles) (right).

\section{TESTS OF ISOTROPY}

Here we use the tests put forward by Briggs [5]. The test statistics are the following:

$$
M_{N}=\frac{1}{N} \sum_{i=1}^{N}\left[\begin{array}{ccc}
x_{i} x_{i} & x_{i} y_{i} & x_{i} z_{i} \\
y_{i} x_{i} & y_{i} y_{i} & y_{i} z_{i} \\
z_{i} x_{i} & z_{i} y_{i} & z_{i} z_{i}
\end{array}\right] \quad R=\sum_{i=1}^{N} \mathbf{r}_{\mathbf{i}} \quad B=\frac{15 N}{2} \sum_{k=1}^{3}\left(\lambda_{k}-\frac{1}{3}\right)^{2}
$$

Here $N$ is the number of elements, $x_{i}, y_{i}$ and $z_{i}$ are the Cartesian coordinates of the bursts on the unit sphere, $r_{i}$ is the unit vector pointing to the bursts and $\lambda_{k}$ are the eigenvalues of $M_{N}\left(\lambda_{1} \geq \lambda_{2} \geq \lambda_{3}\right)$. These statistics are independent of the coordinate system. $R$ is the Rayleigh and $B$ is the Bingham statistic. $W$ is the Watson statistic, related simply to $R$ as $M=3 R^{2} / N$.

\section{RESULTS}

We calculated the test statistic values for the actual observed distribution of the Swift bursts. Afterwards we simulate 1000 catalogues according to the exposure function for the different populations and calculate the mentioned statistics. We find the short and long population are distributed isotropically (p-values from 0.29 to 0.694 for the hypothesis of isotropic distribution). The intermediate population however, shows a marked anisotropy (p-values from 0.038 to 0.072). (see Table 1.) In the distribution of the intermediate duration group we see a dearth of bursts in the lower left part of the sky-distribution.

\section{ACKNOWLEDGMENTS}

This work was supported by OTKA grant K077795, by OTKA/NKTH A08-77719 and A08-77815 grants (Z.B.), by the GAČR grant No. P209/10/0734 (A.M.), by the Research Program MSM0021620860 of the Ministry of Education of the Czech Republic (A.M.) and by a Bolyai Scholarship (I.H.). 
TABLE 1. The different statistics' value for the three groups with $p$-values showing the probability of the measured value occurring by chance based on $1000 \mathrm{MC}$ simulations.

\begin{tabular}{ccccc}
\hline group & $N$ & Bingham $(p$-value $)$ & Rayleigh $(p$-value $)$ & $M_{N}$ eigenv. $\lambda_{1}(p$-value $)$ \\
\hline short & 31 & $78.6(0.644)$ & $5.6(0.409)$ & $0.387(0.553)$ \\
interm. & 46 & $123.8(\mathbf{0 . 0 3 8})$ & $10.4(\mathbf{0 . 0 8 9})$ & $0.441(\mathbf{0 . 0 7 2})$ \\
long & 331 & $831.5(0.694)$ & $23.2(0.290)$ & $0.364(0.645)$ \\
\hline
\end{tabular}
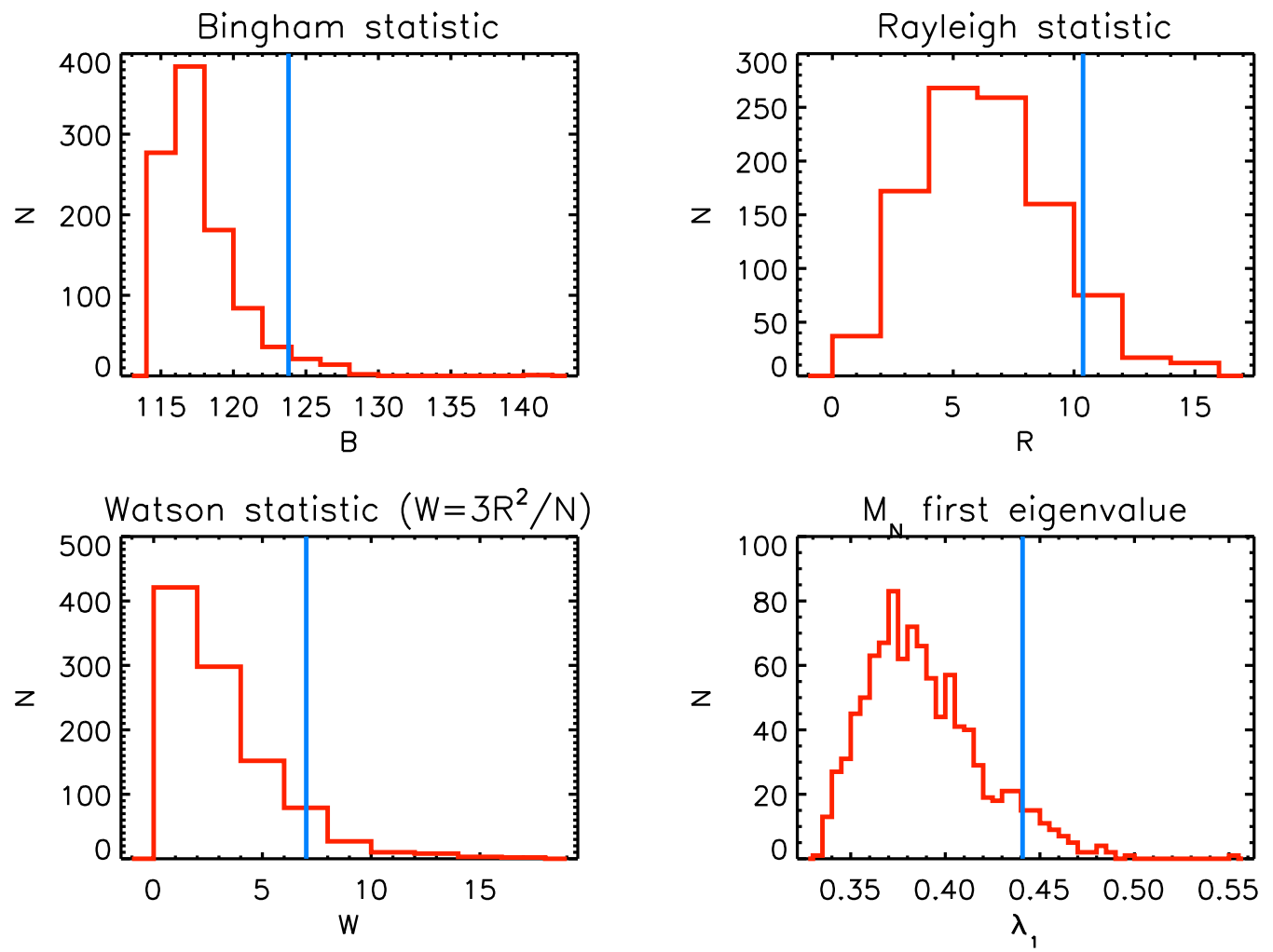

FIGURE 2. The distribution of $1000 \mathrm{MC}$ simulated values of the four statistics for the intermediate-very soft group. The vertical lines mark the values of the measured data statistic.

\section{REFERENCES}

1. P. Veres, Z. Bagoly, I. Horváth, A. Mészáros, and L. G. Balázs, Astrophysical Journal 725, 1955 (2010), 1010.2087.

2. Brock, M. N. et al., "BATSE's sky sensitivity map," in AIP Conference Series, edited by W. S. Paciesas \& G. J. Fishman, 1992, vol. 265, pp. 399-403.

3. Górski, K. M. et al., Astrophysical Journal 622, 759-771 (2005), arXiv : astro-ph/ 0409513.

4. Tueller J. et al. , Astrophysical Journal Supplement Series 186, 378-405 (2010), 0903 . 3037.

5. M. S. Briggs, Astrophysical Journal 407, 126-134 (1993). 